\title{
Estrategias comunicativas desde el ciberfemismo: @Feminismosmad y la cifusión en Twitter de la Huelga del 8M en Madrid*
}

\author{
Dafne Calvo** \\ María Díez-Garrido*** \\ Noel Bandera López****
}

\section{Resumen}

Tanto desde el ámbito académico como desde el movimiento feminista se discute el alcance y la relevancia de las nuevas herramientas digitales para la subversión de la agenda, el cambio en los sistemas políticos occidentales y, en suma, el fin de las desigualdades entre géneros (Zafra, 2011; Núñez Puente, 2011). Mediante un análisis cuantitativo y entrevistas semiestructuradas, estudiamos el uso de Twitter por parte de @FeminismosMad. Este perfil se enfocó en explicar la Huelga feminista, así como en motivar la participación de la ciudadanía en las diferentes actividades organizadas durante el proceso del 8M.

Palabras clave: $8 \mathrm{M}$, Huelga Feminista, Feminismo, ciberfeminismo, Twitter.

* Recibido el 15 de julio de 2018, aceptado el 06 de mayo de 2020. Esta investigación se ha realizado en el marco del proyecto "Estrategias, agendas y discursos en las cibercampañas electorales: medios de comunicación y ciudadanos" (CSO2016-77331-C21-R). En este artículo, la primera autora se ha encargado de la correspondencia con la revista, de realizar la revisión teórica, las entrevistas en profundidad y las conclusiones. La segunda autora ha adaptado la parte metodológica y participado en el análisis de contenido. El tercer autor ha participado en el análisis de contenido y redactado los resultados.

**Investigadora predoctoral en Comunicación en el Departamento de Historia Moderna, Contemporánea y de América, Periodismo y Comunicación Audiovisual y Publicidad. Universidad de Valladolid, Valladolid, España. dafne.calvo@uva.es / http://orcid.org/00000003-0659-6792.

*** Investigadora postdoctoral en Comunicación en el Departamento de Historia Moderna, Contemporánea y de América, Periodismo y Comunicación Audiovisual y Publicidad. Universidad de Valladolid, Valladolid, España. maria.diez.garrido@uva.es / http://orcid.org/0000-0002-5430-7708.

**** Investigador predoctoral en el Instituto de Derechos Humanos. Universitat de València, València, España. noel.bandera-lopez@uv.es / http://orcid.org/0000-0001-7142-0174. 
Communicative Strategies from Cyberfemism: @Feminismosmad and the Twitter Diffusion of the $8 \mathrm{~m}$ Strike in Madrid

\begin{abstract}
The scope and relevance of new digital tools for subverting the dominant agenda, changing Western political systems and ending gender inequalities are discussed from an academic perspective and that of the feminist movement (Zafra, 2011, Núñez Puente, 2011). Through a quantitative analysis and semi-structured interviews, we studied the use of Twitter by @FeminismosMad. The profile focused on explaining the Feminist Strike, and how citizen participation was motivated in different activities organized during the $8 \mathrm{M}$ process.
\end{abstract}

Keywords: $8 \mathrm{M}$, Feminist Strike, Feminism, Cyberfeminism, Twitter. 


\section{Introducción}

El 8 de marzo de 2018, Día Internacional de la Mujer, se celebró por primera vez en España una Huelga feminista, que convocó a decenas de miles de mujeres en movilizaciones a lo largo de todo el territorio. Bajo la proclama de «Si nosotras paramos, el mundo se para», la Comisión 8 de marzo, organizadora del evento, promovió durante los meses previos una huelga que abarcó el plano laboral, de cuidados, de consumo y estudiantil. Las imágenes y relatos de las movilizaciones en ciudades como Barcelona, Zaragoza o Madrid se difundieron a nivel internacional y medios de comunicación extranjeros publicaron titulares como: «Spain kicks off International Women's Day with a strike» (The Washintong Post)' "More than $5 \mathrm{~m}$ join Spain's 'feminist strike', unions say» (The Guardian)"; «En Espagne, les femmes font grève pour "arrêter le monde" "(Le Monde) $)^{3}$ o "Mulheres fazem greve inédita na Espanha» (O dia $)^{4}$. En la capital del Estado se contabilizaron hasta 170.000 participantes en la manifestación según la Delegación del Gobierno y 600.000 según las organizadoras de esta 5 .

El presente repertorio de acciones feministas se enmarca en un nuevo escenario caracterizado por el desarrollo y expansión creciente, a partir de los años noventa, de los dispositivos de comunicación digital de uso particular, de Internet y, por extensión, de redes sociales como Twitter o Facebook (Juris, 2012; Castells, 2012). Las manifestaciones de activismo feminista

\footnotetext{
${ }^{1}$ Información extraída de: https://www.washingtonpost.com/video/world/spain-kicks-offinternational-womens-day-with-a-strike/2018/03/08/ab5032a2-2287-11e8-946c-

$9420060 \mathrm{cb} 7 \mathrm{bd}$ _video.html?noredirect $=$ on\&utm_term $=.7 f 9612980 e 35$ - acceso el $02 \mathrm{sep}$ 2020.

2 Información extraída de: https://www.theguardian. com/world/2018/mar/08/spanishwomen-give-up-work-for-a-day-in-first-feminist-strike - acceso el 03 sep 2020.

3 Información extraída de: https://www.lemonde.fr/europe/article/2018/03/08/en-espagneles-femmes-font-greve-pour-arreter-le-monde_5267305_3214.html - acceso el 03 sep 2020.

4 Información extraída de: https://odia.ig.com.br/mundo-e-ciencia/2018/03/5520340mulheres-fazem-greve-inedita-na-espanha.html - acceso el 04 sep 2020.

${ }^{5}$ Información extraída de: https:/www.elconfidencial.com/espana/2018-03-09/8-m-historicomillones-feminismo-partidos-politicos_1532930/ - acceso el 02 sep 2020.
} 
mediadas tecnológicamente generan nuevos espacios y estrategias para la lucha contra las desigualdades entre géneros, donde la relación entre el entorno digital y los espacios tradicionales de protesta demuestra una diversidad de formas de acción política que merecen ser estudiadas (Núñez Puente, 2011a; Núñez Puente, Vázquez Cupeiro, y Fernández Romero, 2016).

Por ello, tanto el ámbito académico como el movimiento feminista analizan la relación de las mujeres con la tecnología y las opciones para que estas primeras puedan utilizar los instrumentos que propone el ciberespacio para cercenar las desigualdades entre géneros a través de las prácticas de ciberactivismo y, de forma más específica, establecer un discurso propio y superador del dominante en los medios de comunicación masivos (Zafra, 2011; Núnez Puente, 2011b). Si bien el estudio de los medios sociales resulta, por su complejidad, un reto para la investigación social, este en buena medida se ha basado en estudios cualitativo, de forma que el uso que las propias organizaciones realizan de estas plataformas electrónicas aún permanece inexplorado (Edwards, Philip y Gerrard, 2019).

En este trabajo tomamos en consideración tanto el contenido de los mensajes en redes sociales como el propio trabajo interno del movimiento feminista, a fin de conocer las estrategias de comunicación digital llevadas a cabo por la Asamblea organizadora de las movilizaciones del 8 de marzo en el caso específico de Madrid y desde su cuenta de Twitter, Feminismos Madrid (@FeminismosMad) ${ }^{6}$, que centralizaba la difusión online de las acciones llevadas a cabo por el movimiento feminista durante la jornada del 8M y los meses previos en Madrid, los barrios de la ciudad y otras localidades de esta región.

A través de la entrevista semi-estructurada a las responsables de la Comisión de comunicación y del análisis de contenido de los mensajes publicados en la red de microblogging, estudiamos los objetivos de la comunicación digital, la difusión en redes de las protestas y las actividades organizativas de la Huelga, así como su interacción y creación de lazos con la ciudadanía, las instituciones

${ }^{6}$ Véase: https://twitter.com/FeminismosMad - acceso el 02 sep 2020. 
políticas y otros movimientos sociales. Y así, en suma, tratamos de reflexionar sobre el uso que desde el colectivo realizaron de Internet y sobre las capacidades del ciberespacio para colaborar en las acciones de protesta encaminadas a situar el feminismo como una cuestión central en la esfera pública contemporánea.

\section{Movimientos sociales, tecnología y prácticas feministas en Red}

Las dos primeras décadas del siglo XXI han sido escenario de nuevas manifestaciones de acción colectiva que han utilizado la Red como una nueva herramienta para la organización y difusión a través de las protestas masivas contra la deslegitimación de los sistemas políticos democráticos y a favor de nuevas formas de participación de la ciudadanía en democracia (Juris, 2012; Juris, Pereira, y Feixa, 2012; McChesney, 2015). Sucesos acontecidos a nivel global tales como la Primavera Árabe, Occupy Wall Street o el $15 \mathrm{M}$ español han sido analizados desde diversas disciplinas para conocer la relevancia y el alcance que el ciberespacio y las herramientas digitales presentan para organizarse a nivel interno, movilizar a la población, comunicar sus reivindicaciones y generar nuevas estrategias de acción colectiva diferentes a las identificadas en la acción política tradicional (Sandoval-Almazan y Gil-García, 2014; Casero-Ripollés, 2015).

De forma general, la expansión de Internet y su utilización por parte de la ciudadanía han supuesto la manifestación de las prácticas comunicativas de forma automediada, descentralizada y autónoma, sin la dependencia de los medios de comunicación de masas -controlados, por otra parte, por los agentes gubernamentales y económicos- y, por extensión, con la capacidad para encuadrar su discurso y generar significados políticos propios (Castells, 2009, 2012; Cammaerts, 2012). Al mismo tiempo, y aunque se haya descrito a las redes sociales como eficaces para el establecimiento inverso de una agenda concreta, se sigue reflexionando sobre la dependencia de los movimientos de la televisión, radio y prensa como vías de acceso a la población no conectada (Micó y Casero-Ripollés, 2014; Sandoval-Almazan y GilGarcía, 2014; Casero-Ripollés, 2015). 
Diversas investigaciones académicas advierten de la necesidad de seguir analizando la relevancia de ocupar el espacio offline para la consecución de los objetivos políticos de los movimientos, así como de comprender las posibles limitaciones de la participación en el ciberespacio en términos de alcance político. También proponen debatir sobre el mismo uso de redes sociales corporativas -tales como Facebook o Twitter- que atienden a intereses empresariales antes que a los propios de las protestas, dado que se encuentran enmarcadas dentro del contexto capitalista y globalizado de las sociedades donde se producen (Juris, 2012; Castells, 2012; Cornelissen, Karelaia y Soyer, 2013).

Por otro lado, desde que el pensamiento moderno político desarrolló el concepto de ciudadanía como un ideal dirigido a la emancipación del sujeto en el ámbito político, diferentes minorías y grupos oprimidos han intentado ser descritos e incluidos dentro de esta categoría (Young, 1996). Estos movimientos, aun con un recorrido histórico previo, resurgen a finales del siglo XX con el establecimiento de unas condiciones sociales y una cultura política para presentar nuevas formas de acción y organización colectiva de interés para su estudio desde el ámbito académico (De Miguel Álvarez, 2003).

Desde los años noventa hasta la actualidad, el movimiento feminista recoge el legado político y sociológico de sus manifestaciones anteriores para proponer nuevas teorías y críticas sociales desde las teorías queer, posmodernistas y poscoloniales (Mondé, 2018; Wajcman, 2009). Continúa desarrollando un conjunto de acciones orientadas a la generación de una agenda política, la subversión de los discursos hegemónicos y el surgimiento de nuevas prácticas para la movilización y la acción política (Light, 1995; Valcárcel, 2001; Pavan y Mainardi, 2018; BanetWeiser y Miltner, 2019).

A la presencia en torno a páginas web y proyectos netamente feministas en la esfera online se unen las acciones enfocadas a la incidencia política en espacios exógenos al propio movimiento, tales como la publicación de mensajes, viralización de contenidos y debate en redes sociales como Facebook o Twitter 
o, incluso, la publicación de entradas en Wikipedia (Sánchez-Duarte y Fernández-Romero, 2017).

La trascendencia de campañas y movilizaciones feministas es internacional y se ha manifestado a través de diversidad de modos y lugares: la etiqueta \#NiUnaMenos en redes sociales acompañó a las manifestaciones contra los feminicidios en Argentina (Chenou y Cepeda-Másmela, 2019); Italia empleó la misma premisa (Non Una Di Meno) en la celebración del 8 de marzo de 2017 (Pavan y Mainardi, 2018); el lema \#WhyIStayed sirvió en Estados Unidos para articular un discurso dominante en los medios contra la culpabilización de las víctimas de violencia doméstica (Clark, 2016); en Turquía las redes digitales sirvieron como otra esfera de reivindicación contra la penalización del aborto (Eslen-Ziya, 2013), etc.

En el Estado español, la web Mexicoturismo.org, con apariencia de portal turístico sobre el país latinoamericano, se diseñó para denunciar los feminicidios en Ciudad Juárez y la campaña «Yo también quiero ser excomulgado/a» nació como respuesta a una advertencia de la Iglesia nicaragüense, que se oponía al aborto de una niña de nueve años embarazada a causa de una violación (Boix, 2006). Red Feminista, por su parte, surgió como una extensión del discurso y objetivos de varios grupos que operaban de forma offline bajo la consideración de la justicia como patriarcal y la significación de la violencia de género como equiparable a un acto terrorista (Núñez Puente, 2011a, 2011b). El movimiento español también promovió el uso de etiquetas durante las protestas del 8M de 2017 (Núñez Puente, D’Antonio Maceiras y Fernández Romero, 2019).

Estas acciones constituyen una articulación de las reivindicaciones del propio movimiento feminista en el espacio online, que describen una diversidad de relaciones con las formas tradicionales de movilización y protesta, bien sea porque la Red se convierte exclusivamente en una forma de publicidad de las acciones en la esfera offline, porque algunos colectivos limiten su funcionamiento al ciberespacio o porque manifiesten formas combinadas de acción colectiva, donde las prácticas en la esfera 
digital y la presencial se hibridan y retroalimentan (Núñez Puente, 2011a; Núñez Puente, Vázquez Cupeiro, y Fernández Romero, 2016).

La construcción y desarrollo de estos nuevos espacios y repertorios de acción en la Web suponen la generación de expectativas para la acción política del propio movimiento. Pero a la vez, resulta esencial reflexionar sobre el alcance real de estos nuevos espacios de lucha contra las desigualdades entre géneros y si estos suponen elementos reiterativos limitados al apoyo de la praxis feminista tradicional (Núñez Puente, Vázquez Cupeiro y Fernández Romero, 2016). Diferentes interpretaciones feministas advierten que de forma histórica los avances tecnológicos han sido modelados por hombres tanto en su forma como en su contenido. $\mathrm{Y}$ de esta manera, en el uso de herramientas digitales $-\mathrm{y}$ extensivamente de Internet- influyen las jerarquías tradicionales del mundo offline (Light, 1995; Wajcman, 2007, 2009). Manifestaciones de ello son, según van Zoonen (2002), la existencia del acoso sexual en redes, la distribución de pornografía o las opiniones sobre derechos civiles desde la extrema derecha que afectan directamente a la mujer.

En corto, el poder y privilegio masculinos han reaccionado con el desarrollo de movimientos antifeministas $y$ fundamentalmente misóginos que se movilizan alrededor de temas específicos, como la violencia doméstica contra hombres, la custodia de los hijos, el divorcio o la feminización de la educación. La migración de estos grupos a los medios y redes sociales ha supuesto un relevante cambio en sus estrategias, preocupaciones y modos comunicativos del feminismo (Banet-Weiser, 2018; Ging, 2017, 2019). Campañas en redes como \#masculinitysoFragile se originaron, de hecho, con la intención de apuntar hacia formas de masculinidad tóxica y comportamientos sexistas tanto dentro como fuera de la Red (Banet-Weiser, 2018; Banet-Weiser y Miltner, 2019).

Al mismo tiempo, el propio empoderamiento tecnológico se encuentra afectado por los mecanismos sociales que suponen nuevas formas de exclusión en la Web - $\mathrm{y}$ con ello, mayor dificultad para la conquista cultural y política online-, no solo en torno al género, sino también al origen étnico, la condición social 
o el nivel educativo (Rúdólfsdóttir y Jóhannsdóttir, 2018; Orgad y Gill, 2019). La emancipación y empoderamiento de las tecnologías de la información a través del autodidactismo, la concienciación de una identidad propia en Red y el análisis crítico de las herramientas y formas de producción de información son estrategias y recursos que desde posiciones feministas plantean subvertir las desigualdades estructurales de una sociedad crecientemente conectada (Haraway, 1991; Zafra, 2001).

Por todo ello, autoras como Gill (2008) declaran una tarea urgente explorar la trabazón entre las experiencias de subjetividad y las cambiantes formas de organización política, relaciones sociales y prácticas culturales. Internet se propone entonces como un instrumento para el ciberactivismo feminista y a la vez como un espacio de debate sobre las desigualdades estructurales que se producen de forma histórica entre géneros.

\section{Metodología}

Esta investigación pretende aproximarse a los procesos de reivindicación y protesta feministas en el ciberespacio a través de una red social y un evento específico: el uso de Twitter para la difusión, movilización y organización de la Huelga feminista del 8M de 2018 en el Estado español. Más concretamente, nos centramos en el caso de Madrid, por ser una de las ciudades con mayor seguimiento de la manifestación durante la jornada de Huelga. Como se indica en la introducción, en la capital del Estado se reunieron 170.000 personas en datos de la Delegación del Gobierno y unas 600.000 según las organizadoras ${ }^{7}$. Propusimos, así, los siguientes objetivos: 1) conocer las estrategias de comunicación digital llevadas a cabo por la comisión promotora de las movilizaciones del 8 de marzo en Twitter, 2) describir la organización y planificación interna de la comunicación digital a través de este espacio y, por último, 3)

\footnotetext{
7 Información extraída de: https://www.elconfidencial.com/espana/2018-03-09/8-mhistorico-millones-feminismo-partidos-politicos_1532930/ - acceso el 04 sep 2020.
} 
analizar los mensajes o tweets que se difundieron durante la jornada del 8M, así como en los meses previos a esta.

Para cumplir con los objetivos anteriormente mencionados, planteamos una metodología doble, brevemente adelantada en el apartado introductorio. Realizamos un análisis de contenido de los mensajes de la cuenta de Twitter de la organización, Feminismos Madrid (@FeminismosMad), a la que seguían más de 15.000 perfiles en el momento en el que se realizó este análisis, según los indicadores de la propia red social ${ }^{8}$. Posteriormente, planteamos una entrevista en profundidad con dos mujeres participantes de la Asamblea 8 de marzo en Madrid, colectivo desde el cual se planificaron, organizaron y ejecutaron las actividades en torno a la Huelga feminista del 8M, incluida también la difusión de estas a través de Twitter. La metodología utilizada, tanto en su enfoque cuantitativo como desde la vertiente cualitativa, se basa en la desarrollada por Dader y Campos (2017) y que autor y autora aplicaron a las cibercampañas electorales españolas durante la convocatoria de 2015.

En un primero paso, por tanto, analizamos el tipo de mensajes difundidos por la Asamblea Feminista de Madrid en su cuenta de Twitter. De nuevo tomamos como referencia la propuesta metodológica de Dader y Campos (2017), pero adaptada a las características estructurales y circunstanciales de este movimiento social y evento concretos. El periodo de análisis estudiado se encuentra entre el 1 de septiembre de 2017 hasta el 22 de marzo de 2018 (dos semanas después del 8 de marzo, por tanto). Esta periodización responde al interés por describir el desarrollo y evolución del uso de Twitter desde la preparación de la Huelga hasta las semanas posteriores a esta.

Durante esta etapa, @FeminismosMad publicó 1.963 tweets, de los cuales analizamos 623, a través de un muestreo sistemático que nos permitiera seleccionar mensajes de todas las fases de la organización de la Huelga y además de forma proporcional a la frecuencia de publicación en cada periodo trascurrido en el marco temporal de la investigación. Un codificador y una codificadora se

${ }^{8}$ Información extraída de: https://twitter.com/FeminismosMad - acceso el 03 sep 2020. 
encargaron de ejecutar el análisis de contenido, previo pretest para resolver las diferencias en la interpretación de los mensajes. Cada tweet funcionó como una unidad de análisis, a la cual le aplicamos 21 variables, que podemos sintetizar de la siguiente forma: 1) Características del mensaje; 2) Contenido de los mensajes y 3) Marcos informativos (framing).

En una segunda fase, con el objetivo de conocer la gestión interna de la Asamblea 8M de Madrid, realizamos una entrevista en profundidad a la responsable de redes dentro de la Comisión de Comunicación y encargada de la cuenta de Twitter y a una participante de la Comisión de Comunicación y la Comisión de Prensa del 8M Madrid. El cuestionario se realizó el 19 de mayo en Madrid. Se trató de una entrevista semi-estructurada, con 62 preguntas divididas en cinco bloques, a saber: 1) Organización interna; 2) Uso de Internet; 3) Estrategia en la práctica de mensajes y gestión de redes; 4) Medios de comunicación y 5) Resultados del análisis de contenido.

Las entrevistas en profundidad proporcionaron una gran riqueza de detalles y precisión en las cuestiones relacionadas con los objetivos del estudio, y facilitaron comprender aspectos sobre el proceso de comunicación del $8 \mathrm{M}$ que a través de otro método resultan complejos de averiguar (Wimmer y Dominick, 1996), a saber: perspectivas internas de la asamblea, sus circunstancias organizativas, sus objetivos comunicativos, etc. El análisis de contenido, por su parte, ofrecía datos sistematizados para extraer conclusiones sustentadas en procedimientos de validación numéricos (Igartua, 2006). Por ello, la aplicación de esta primera perspectiva cuantitativa se complementaba con la segunda, cualitativa, y su combinación permitía reducir las limitaciones de la investigación (Delgado y Gutiérrez, 1995), así como atender a las perspectivas internas y externas del fenómeno social analizado. 


\section{Resultados}

\subsection{Organización interna de la Asamblea 8M}

La convocatoria del 8M de 2018 nace de un escenario significativamente diferente al de años anteriores, pues por primera vez el movimiento feminista convocaba una acción política "absolutamente novedosa», en palabras de las propias entrevistadas, quienes consideraban uno de sus objetivos principales a nivel comunicativo explicar el significado de esta forma de acción política. Ello supuso no solo un aumento cuantitativo del número de mujeres participantes en las Asambleas - de 20 a 500, según la organización-, sino un recorrido más prolongado para la organización y preparación de la jornada, pues si bien hasta 2016 esta comenzaba a reunirse a partir de enero del mismo año para organizar la manifestación, la gestación de la Huelga de 2018 data de abril de 2017.

Así, aunque la propia Asamblea del 8M de Madrid surge entre la década de los setenta y ochenta del siglo anterior, también la actividad comunicativa de esta estuvo, a diferencia de experiencias previas, más organizada en su estructura. A cada tarea se asignaba un grupo de trabajo concreto para la diversificación de los objetivos, ya que en 2017 surgieron nuevos grupos de trabajo, como la Comisión de Accesibilidad, la de Acciones o la de Prensa.

La tarea de difusión en redes sociales se concretó dentro de Comunicación, donde, grosso modo, cada una de las integrantes dedicadas a las estrategias de movilización online tuvo asignada una red social concreta: Facebook, YouTube, Instagram y Twitter. Por otro lado, las cuentas utilizadas en las diferentes plataformas no se abrieron exprofeso para la Huelga, sino que, como la propia Asamblea, se habían creado con anterioridad. En el caso concreto de Feminismos Madrid (@FeminismosMad), el perfil data del 15 de febrero de 2017, de manera que el trabajo de aumento de seguidoras y seguidores fue facilitado por el propio recorrido previo de la cuenta, que ya había servido a los fines de movilización de la manifestación del 8 de marzo de 2017. 
En su mayoría, las participantes respondían a un perfil joven, de «nativas digitales» — como se autodenominaban ellas mismas- que ya habían participado en las tareas comunicativas digitales en otros espacios, como asociaciones estudiantiles o la preparación del 8M durante 2016. Sin embargo, las responsables de medios no eran, de forma general, profesionales del ámbito comunicativo, sino que pertenecían a otras disciplinas como filosofía o historia. En este sentido, las entrevistadas señalaban no haber realizado ningún adiestramiento en el uso de redes sociales debido a su edad y experiencia previa con ellas, si bien reconocían haber planeado un taller en gestión de la difusión online que por cuestiones organizativas no pudieron finalmente llevar a cabo.

La comunicación interna de esta área de trabajo fue esencialmente online, a través del grupo de WhatsApp creado al respecto y, eventualmente, de Telegram y Skype. Desde ese entorno online se planificaban los contenidos, que posteriormente se aplicaban a la actividad diaria en redes sociales. Este tipo de coordinación difiere de la de la propia Asamblea, la cual se reunía el día 8 de cada mes de forma presencial para discutir las cuestiones más relevantes del trabajo de las Comisiones.

Era en estos espacios donde las estrategias comunicativas, también las del ámbito digital, se consensuaban con todas las participantes en las reuniones - abiertas para todas las mujeres que desearan formar parte de ellas-. Fue en este contexto donde validaron el argumentario creado por la Comisión de Contenidos y dispuesto posteriormente de forma pública, a partir del cual se generaban los mensajes publicados en Twitter. Era también en estos espacios donde la Asamblea en su conjunto se informaba y reconocía la propia actividad en redes. De esta manera, aunque las decisiones orgánicas y políticas relevantes se debatieron de manera offline, el trabajo diario, que requería una capacidad de improvisación y reacción más inmediatas, fue autónomo y a través de canales de interacción online. 


\subsection{Características de los mensajes en Twitter}

Las participantes de la Comisión de Comunicación reconocían haber realizado una difusión en redes improvisada, más dependiente de las circunstancias y necesidades que iban percibiendo durante las fases de preparación y desarrollo de la Huelga. De la misma forma, no contaban con un libro de estilo, si bien la interacción inmediata a través del grupo de WhatsApp facilitaba que las activistas consensuaran criterios de publicación: sucedía, por ejemplo, en el caso de los hashtags, donde debatían con el resto de las compañeras aquellos que se publicarían en Twitter. El 58,75\% de publicaciones de @FeminismosMad hicieron uso de este recurso, con una media de 1,55 etiquetas por cada uno de los 366 mensajes que contaban con ella $-0,91$ hashtag por tweet si contabilizamos la muestra total-, tales como \#PerreaHaciaLaHuelga o \#VengoaHablardeMiHuelga, así como otros más generales, como \#HaciaLaHuelgaFeminista o \#Huelga8M.

La utilización de los hashtags, según las activistas, presentaba el objetivo de viralizar las publicaciones y obtener más visibilidad en esta red social, a través esencialmente del posicionamiento como un Trending Topic. La utilización de retweets seguía también esta estrategia y se vinculaba a la publicación de las etiquetas diseñadas por la propia cuenta: «Cuando usaban el hashtag que proponíamos, hacíamos retweets para fomentar y motivar que publicaran nuestro hashtag y que así este moviera más». La distribución de las autorías de los mensajes analizados en la muestra seleccionada fue la siguiente: un $65 \%$ eran tweets propios, un $28,6 \%$ retweets y un $6,4 \%$ tweets incrustados o comentados. Casi todos los incrustados y compartidos citaban a cuentas hermanas de la de Feminismos Madrid, como las comisiones promotoras en otros territorios $(10,1 \%)$, a otros grupos feministas $(9,1 \%)$ y a cuentas personales que se autodefinían como feministas (4,1\%). Al mismo tiempo, destaca la baja presencia de retweets a medios de comunicación $(1,77 \%)$ y a algún tipo de organización sindicalista $(0,6 \%)$. 
En esta misma línea, los mensajes con menciones, que representan un $23,43 \%$ del total, no solían referenciar a partidos políticos o sindicatos —más allá de algunos reproches a organizaciones concretas-. No apreciamos una relación relevante con organizaciones tradicionales que pudieran tener posicionamientos en teoría cercanos, sino que los vínculos más fuertes se construyeron con otros colectivos, como la Plataforma de Afectados por la Hipoteca (PAH), los Yayoflautas o el Movimiento 12N (Movimiento de Acción Política Antirracista).

La interacción con partidos políticos y sindicatos resultó, de hecho, una de las cuestiones de debate interno en la Comisión de comunicación del 8M Madrid, pues su relación con estas organizaciones variaba dependiendo de la ideología, la estructura y la perspectiva feminista de estas. Si bien en principio sí consideraron viralizar y mencionar los mensajes de estas cuentas, finalmente consensuaron el criterio de solamente difundir aquellos eventos organizados donde participara alguna de las participantes en la Asamblea del 8M, con la intención, principalmente, de significar la Huelga como una acción política organizada desde este activismo y evitar la apropiación de esta por parte de grupos exógenos:

Al final el movimiento feminista es muy autónomo y nos alejamos de todo lo que fuera más organizado, más cercano a la institución. Y además, tener relación con los movimientos sociales era lo más importante y cuando se crean las cuentas de los propios barrios, pues interactuábamos con estos. 
Tabla 1: Características básicas de los mensajes.

\begin{tabular}{|c|c|}
\hline Variable & Resultados \\
\hline Autoría & $\begin{array}{r}405=65,01 \%=\text { Tweet propio } \\
178=28,57 \%=\text { Retweet } \\
40=6,42 \%=\text { Tweet incrustado y } \\
\text { comentado }\end{array}$ \\
\hline $\begin{array}{c}\text { Autoría del retweet } \\
\text { (solo en caso de que sea retweet o tweet } \\
\text { incrustado) }\end{array}$ & $\begin{array}{r}408=65,49 \%=\text { No aplica } \\
63=10,11 \%=\text { Cuentas hermanas } \\
57=9,15 \%=\text { Cuenta de un grupo } \\
\text { feminista } \\
26=4,17 \%=\text { Cuenta personal } \\
\text { autodefinida como feminista } \\
11=1,77 \%=\text { Medio de comunicación } \\
4=0,64 \%=\text { Sindicato } \\
54=8,67 \%=\text { Otros }\end{array}$ \\
\hline Presencia de texto & $\begin{aligned} 620 & =99,52 \%=\text { Sí } \\
3 & =0,48 \%=\text { No }\end{aligned}$ \\
\hline Acompañamiento de una fotografía & $\begin{array}{c}385=61,8 \%=\text { Sí } \\
238=38,2 \%=\text { No }\end{array}$ \\
\hline Acompañamiento de un vídeo & $\begin{array}{r}43=6,9 \%=\text { Sí } \\
580=93,1 \%=\text { No }\end{array}$ \\
\hline Presencia de contenido de enlaces & $\begin{array}{c}79=12,68 \%=\text { Sí } \\
544=87,32 \%=\text { No }\end{array}$ \\
\hline Presencia en un hilo & $\begin{array}{c}112=17,98 \%=\text { Sí } \\
511=82,02 \%=\text { No }\end{array}$ \\
\hline Presencia de menciones & $\begin{array}{c}146=23,43 \%=\text { Sí } \\
477=76,57 \%=\text { No }\end{array}$ \\
\hline Presencia de hashtags & $\begin{array}{c}366=58,75 \%=\text { Sí } \\
257=41,25 \%=\text { No }\end{array}$ \\
\hline
\end{tabular}

Fuente: elaboración propia. 
En términos de personalización, el $84,1 \%$ de los mensajes no estaban dirigidos a ninguna persona ni colectivo concreto. Con relación al tono elegido, un $55,7 \%$ de los textos fueron etiquetados como formales, un $15,09 \%$ como informales y un $27,93 \%$ como híbridos. A través de la interacción en redes sociales, las activistas percibieron cómo a cada uno de los canales utilizados por la Asamblea 8M de Madrid le correspondían unos objetivos, dinámicas y requerimientos propios, dependientes de las y los internautas que se encontraban en cada uno de los espacios digitales:

En Twitter nos llegaban preguntas sobre nuestro
posicionamiento, preguntas como más de contenido
político. En Instagram nos escribían chicas diciendo que el
$8 \mathrm{M}$ tenían examen, que cómo se lo podían decir a la
profesora para no acudir. Y en Facebook, pues eran
preguntas de un grupo feminista en Moratalaz, por ejemplo,
al que querían darle difusión. Era como muy evidente qué
perfiles hay. En Twitter eran periodistas y gente politizada,
gente del activismo, de movimientos sociales.

También con la intención de explicar las consideraciones correspondientes en Twitter, la Comisión de comunicación comenzó a publicar hilos [Ilustración 1]. Esta nueva funcionalidad de la red social, que aparece en un 17,98\% de las publicaciones analizadas, fue considerada de forma positiva por las participantes de la Asamblea 8M, pues permitía agrupar la información, desarrollarla, ordenarla y conseguir la viralización de un conjunto más amplio de mensajes cuando el primero de ellos obtuviera un retweet. Las activistas manifestaron, además, una vocación de conocer y aprovechar los recursos de cada una de las redes sociales empleadas: «Usar las encuestas de Twitter, las instastories, los eventos en Facebook de las asambleas para invitar a la gente...». 
Ilustración 1: Hilo de Twitter (fragmento).



Fuente: @FeminismosMad.

El contenido audiovisual, por su parte, presentaba un interés ilustrativo, especialmente durante el $8 \mathrm{M}$, para difundir las acciones que sucedieron en los diferentes puntos de la capital de Madrid y las otras localidades de la región, incluida la manifestación que en la capital congregó a 600.000 personas. En los meses previos, las fotografías de las Asambleas públicas resultaban un ejercicio de transparencia, así como una invitación a la participación de nuevas asistentes: "Hay gente que no sabe qué es una Asamblea, que nunca ha estado en una o no se atreve a ir sola. El hecho de que te estén contando lo que pasa y cómo es el espacio, es una forma de facilitar a que se anime a venir».

Los tweets contenían vídeos en un $6,9 \%$ de los casos y fotografías o memes en un $38,2 \%$ del total, frente al $12,68 \%$ que 
incorporaba un enlace. Esta significativa preferencia por contenidos subidos a la misma red social $-\mathrm{y}$ no links hacia otras plataformas multimedia- posee al menos dos causas, manifestadas por las propias participantes de la Comisión de Comunicación: en primer lugar, procuraban integrar el contenido multimedia en Twitter, pues de esa forma no era necesario navegar a un sitio externo para consumir ese contenido concreto, como sucede en el caso de la adición de enlaces. Y, además, como adelantamos anteriormente, las fotografías contaban con una especial relevancia para la retransmisión de las acciones de calle y de la actividad en las reuniones previas de la Asamblea.

\subsection{Temática y encuadre de los tweets}

Como exponemos en epígrafes anteriores, las decisiones de la Asamblea se consensuaban en reuniones presenciales y los acuerdos se desarrollaban dentro de los diferentes grupos generados por cada uno de los objetivos y tareas que desarrollaba el movimiento. La Comisión de comunicación creaba su repertorio a partir de un argumentario público definido por la Comisión de contenido y, si surgía alguna duda concreta para la publicación de informaciones y consignas en redes sociales, la coordinación con esta y otras comisiones era fluida y a través de plataformas online, esencialmente WhastApp.

En cuanto al análisis de contenido, comprobamos que en la temática de los tweets se distinguían cuatro ámbitos de relevancia para@FeminismosMad y otros cuatro cuya presencia resulta subalterna [Tabla 2]. Entre este segundo grupo se encontraban las propuestas específicas de partidos o sindicatos $(1,12 \%)$, los encuentros con los medios $(2,41 \%)$, los asuntos políticos no feministas $(3,05 \%)$ y los actos institucionales $(3,37 \%)$. Los temas que sí copaban los mensajes de la cuenta eran las propuestas concretas del 8M (11,56\%), las peticiones expresas de realizar huelga $(16,85 \%) y$, ante todo, las proclamas feministas generales $(22,15 \%)$ y los actos de corte cívico-político $(23,76 \%)$, en especial las asambleas organizadas y otros eventos que organizaron para la difusión offline, como la presentación de esta a través de los 
conciertos del Eventazo o el Día de los mercados. El encuentro con los medios más destacable, por su parte, fue la rueda de prensa de febrero que anunciaba la convocatoria.

Tabla 2: Temática del tweet.

\begin{tabular}{|l|l|}
\multicolumn{1}{|c|}{ Variable } & Resultados \\
\hline Actos de corte cívico-político & $147=23,59 \%$ \\
\hline Proclamas asuntos feministas & $138=22,15 \%$ \\
\hline Petición expresa de realizar la Huelga & $104=16,69 \%$ \\
\hline Propuestas concretas de Asamblea 8M & $72=11,56 \%$ \\
\hline Acto institucional & $21=3,37 \%$ \\
\hline Otros asuntos políticos no feministas & $19=3,05 \%$ \\
\hline Encuentro con los medios & $15=2,41 \%$ \\
\hline Propuestas específicas de partidos y sindicatos & $7=1,12 \%$ \\
\hline Asuntos no mencionados anteriormente & $98=15,73 \%$ \\
\hline Sin asignar & $2=0,32 \%$ \\
\hline Total & $623=100,00 \%$ \\
\hline
\end{tabular}

Fuente: elaboración propia. 
Tabla 3: Encuadre de los mensajes en Twitter

\begin{tabular}{|c|l|}
\hline Variable & \multicolumn{1}{|c|}{ Resultados } \\
\hline Encuadre de apelación emocional & $91=14,61 \%=$ Sí \\
& $532=85,39 \%=$ No \\
Encuadre de juego estratégico & $233=37,4 \%=$ Sí \\
& $390=62,6 \%=$ No \\
\hline Encuadre de posición política & $219=35,15 \%=$ Sí \\
Encuadre logístico-movilizador & $404=64,85 \%=$ No \\
& $366=58,75 \%=$ Sí \\
& $257=41,25 \%=$ No \\
\hline
\end{tabular}

Fuente: elaboración propia

Las participantes de la Comisión de comunicación admitieron que, a pesar de que su intención era la de ejecutar campañas preparadas con suficiente antelación, en la práctica han ido más bien adaptándose a los acontecimientos. Señalaron, además, haberse enfrentado a un reto innovador en el repertorio de acción feminista en el Estado - y a nivel internacional-, como era la presentación de la Huelga, por lo que su trabajo de difusión en redes era exploratorio y la experiencia obtenida en 2018 sirvió para sentar las bases de las estrategias y acciones de movilización online para años posteriores.

Ese contexto, así como la propia naturaleza del movimiento, presentó implicaciones significativas en la línea de actuación discursiva en Twitter y, por tanto, en la temática de sus mensajes. En relación con las proclamas feministas generales y propuestas concretas del $8 \mathrm{M}$, las activistas explicaban que siempre publicaban desde el acuerdo con el resto de sus compañeras: «En esta convocatoria hemos ido a todo lo que suma y a todo lo que sí es consenso y a hablar desde ahí». Una máxima que afectaba también a las preguntas en redes cuando versaban sobre posicionamientos políticos, pues se daba respuesta a las cuestiones donde existiera una conformidad en la Asamblea. 
Al mismo tiempo, consideraban la relevancia de presentar la Huelga, y de la misma forma el nuevo escenario que planteaba. Las entrevistadas eran conscientes de la complejidad de la propuesta de acción colectiva que presentaba su modelo innovador, desarrollado desde cuatro frentes diferentes: estudiantil, laboral, de consumo y de cuidados. La comunicación desplegada en Internet y más específicamente en Twitter debía apelar a estos cuatro vértices y a las implicaciones específicas de la movilización en cada ámbito: «Teníamos que explicarle a la gente qué es esto de que rompemos con la huelga tradicional y en base a eso todo era sacar contenido informativo sobre qué pasa si quieres hacer huelga».

De hecho, las activistas reconocieron que una de las cuestiones mejorables de cara a la difusión digital del $8 \mathrm{M}$ se trataba de la focalización en la explicación de las acciones menos visibilizadas dentro del repertorio de acción colectiva, a saber: la de consumo y especialmente la de cuidados. Percibieron, además, cómo el discurso de la ciudadanía se centraba en el ámbito laboral a pesar de que la cuestión que más debate generaba dentro de la propia asamblea era la de cuidados. Por ello, desde la Comisión de comunicación decidieron enfocar sus esfuerzos concretos en explicar esta última; especialmente, indicaban, cuando los mensajes directos estaban escritos por hombres, para lo que contaban con respuestas tipo a las preguntas que se repetían con mayor frecuencia [Ilustración 2]:

$\mathrm{Ni}$ uno solo [de los hombres] nos preguntó en todo el proceso si podían hacer la [huelga] de cuidados. Teníamos a un montón de mujeres preguntándonos cómo hacer huelga de cuidados y a un montón de hombres preguntándonos si podían hacer la laboral. Hicimos mucho trabajo en poner el foco en la de cuidados porque al final es la más feminista de todas. 
Ilustración 2: Mensaje con proclama feminista dirigido a los hombres.

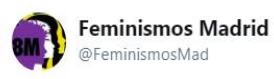

Seguir

Es importante "que los hombres se pregunten por qué se propone esta huelga y qué tienen que hacer para evitar una segunda huelga".

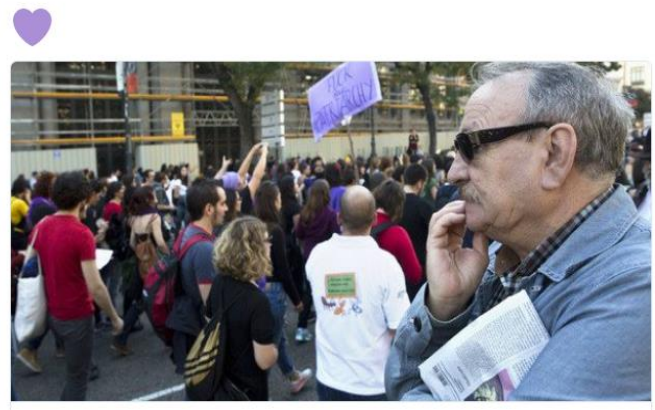

¿Y yo qué tengo que hacer? El papel de los hombres en la huelga del $8 \mathrm{M}$

Fuente: @FeminismosMad.

Por todo ello, a pesar de no interactuar apenas con organizaciones políticas o sindicales, el encuadre político se encontraba relativamente presente en la cuenta @FeminismosMad $(35,15 \%)$ y se situaba cerca del framing estratégico $(37,4 \%)$ [Tabla 3]. En este sentido, las entrevistadas expresaban también cierta inclinación a explicar, desde posiciones más teóricas, las implicaciones del feminismo en la crítica que este realiza de las estructuras de poder y las instituciones sociales y políticas que producen y reproducen las desigualdades entre géneros. Adquieren, para ello, una posición didáctica [llustración 3]. Así, significativamente, la apuesta por posicionamientos que disfruten de suficiente consenso en el movimiento es compatible con el desvelamiento de las conexiones entre capitalismo y patriarcado:

Si decimos que las políticas neoliberales son de por sí machistas, pues entonces lo que estamos diciendo es que el feminismo es anticapitalista y lo estamos diciendo de una forma pedagógica. Entonces, hacemos como un esfuerzo de 
hacer pedagogía con nuestro propio argumentario para que la Huelga tenga un discurso, para que la gente sepa, que haga huelga.

Ilustración 3: Tweet con encuadre político y mención a la representante de un partido.

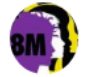

Feminismos Madrid

@FeminismosMad

Seguir

Hola@InesArrimadas, para que no te pillen

en la próxima

Te enviamos las reivindicaciones de la Huelga Feminista:

\section{hacialahuelgafeminista.org/wp-content/upl ...}

\section{El HuffPost @ElHuffPost \\ Críticas a Inés Arrimadas por lo que dijo sobre la huelga feminista en @SextaNocheTV huffingtonpost.es/2018/01/28/cri...}

Fuente: @FeminismosMad.

Los dos encuadres que más se distanciaron en cuanto a su frecuencia de publicación son el emocional —solo un $14,61 \%$ de los mensajes se catalogan así- y el logístico-movilizador, que destaca con un $58,75 \%$ debido a la continua utilización del medio para anunciar e informar sobre los distintos actos feministas que se realizan en Madrid [Tabla 3]. Estos contenidos se encontraban entre los objetivos de la Comisión de comunicación, también en los comienzos del proceso, donde procuraron visibilizar la Asamblea con la intención de que más mujeres acudieran a ella. Solo una vez la movilización comenzó a crecer, las redes sociales centraron sus mensajes en difundir eventos concretos y mejorar la comprensión del escenario del 8M: «Al principio nos dedicábamos más a llamar a la Huelga y posteriormente a explicarla».

Los eventos, protestas y acciones políticas en general también formaron parte del conjunto de contenidos susceptibles de ser difundidos; sin embargo, procuraron no perder en ningún 
caso su vinculación con la movilización del $8 \mathrm{M}$, como sucedió en las campañas del 28 de septiembre (Día de Acción Global por un aborto legal y seguro) o del 25 de noviembre (Día Internacional Contra la Violencia Machista), que siempre se propusieron como jornadas ligadas a la Huelga. Aunque existieron pocas publicaciones con contenido político no relacionado con temas feministas, sí hubo espacio para llamadas a la movilización en este tipo de mensajes [Ilustración 4].

Colectivos como los mencionados anteriormente, La ingobernable, Coca cola en lucha o el movimiento feminista latinoamericano se presentaron como algunos de los referentes políticos y comunicativos de la Comisión, tanto en aspectos más técnicos - en la edición de vídeos, por ejemplo- como por su capacidad para la generación de significados y símbolos políticos de su lucha concreta. Estos movimientos, de los que recibieron apoyo en la difusión de las propuestas y llamadas a la acción política durante el $8 \mathrm{M}$, formaban parte de una red de apoyo generada a través de, entre otras cuestiones, la interacción en Twitter. Durante el proceso de la organización de la Huelga, esta plataforma, a la que describieron como un «nido de activismo», facilitó la manifestación de estos lazos, que las activistas consideraron positivos tanto por las alianzas estratégicas generadas a partir de ellos como por la propia naturaleza interseccional y transversal del movimiento feminista autónomo de Madrid. 
Ilustración 4: Publicación logístico-movilizadora.

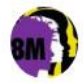

Feminismos Madrid

@FeminismosMad

Seguir

En un rato el Sindicato de Manteros y Lateros

de Madrid darán una rueda de prensa en

@Ecooo_y por la tarde está convocada una concentración \#TodosSomosMame Contra el racismo y la xenofobia \#NingunSerHumanoEsllegal

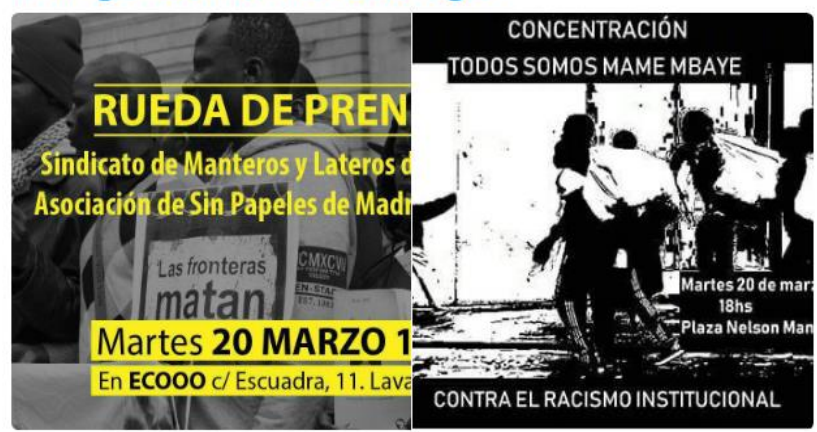

2:16 - 20 mar. 2018

Fuente: @FeminismosMad.

\section{Conclusiones}

El 8 de marzo, Día Internacional de la Mujer, se presenta como una fecha histórica para el movimiento feminista y la efeméride ha sido tradicionalmente empleada para situar en la agenda pública las reivindicaciones y protestas contra la desigualdad entre géneros. Además, desde los años noventa, el activismo feminista español ha generado espacios y formas de protesta en lo offline y online gracias, en este último caso, a la expansión de Internet y de los dispositivos electrónicos de uso personal.

La Huelga de 2018 planteaba una propuesta de acción política inédita dentro de este movimiento, pues se trataba de la primera movilización social de estas características desplegada, además, en cuatro ámbitos diferentes, a saber: el estudiantil, el 
laboral, el de cuidados y el de consumo. A pesar de la existencia previa de un colectivo promotor de las actividades durante el $8 \mathrm{M}$ en años anteriores, las nuevas circunstancias propuestas en 2018 implicaron un aumento significativo del número de participantes - de 20 a 500- y un trabajo mejor organizado con relación a las tareas y áreas más significativas. De forma intuitiva, las responsables de redes conocían el público al que se dirigían en cada uno de los canales desplegados por la Asamblea 8M en Madrid y, en el caso específico de Twitter, utilizaron recursos como las imágenes o videos incrustados, los hilos o el diseño de hashtags propios. Todo ello sin un libro de estilo, sino a través de acuerdos producidos en la mayor parte de los casos en su grupo de WhatsApp.

Contaba, por tanto, con una naturaleza transversal y compleja, desplegada en el ámbito de la movilización para el fomento de la participación en las acciones organizadas en torno al 8M, pero desarrollada también en la esfera discursiva, en tanto existía un esfuerzo declarado por la elaboración de un mensaje contrahegemónico que fuera capaz de articular el sentido político de la efeméride (Valcárcel, 2001; Banet-Weiser y Miltner, 2019).

El enfoque ideológico del movimiento feminista aquí analizado bebe de un marco teórico compartido con autoras como Gill (2008) o Ging (2019), en tanto que se propone entender el feminismo y las subjetividades en su contexto socio-cultural y concretamente respecto al poder neoliberal dominante en la actualidad. Partiendo de esa base, uno de los principales objetivos comunicativos expresados por las entrevistadas, como hemos visto, es el de desvelar las relaciones entre capitalismo y patriarcado e, incluso, la incompatibilidad del sistema económico actual con el feminismo.

Las formas de movilización política propuestas por el movimiento feminista para el $8 \mathrm{M}$ no se circunscriben al ámbito online, sino que la relación entre la esfera presencial y la digital presenta mayor complejidad. Por un lado, las redes sociales se presentan como una de las herramientas a través de las cuales el proceso de organización de la Huelga se manifiesta, bien sea con 
el objetivo de llamar a la participación offline o como una fuente para la explicación y difusión del argumentario y propuestas emanados desde la propia Asamblea.

Pero, además, la propia estructura organizativa del colectivo es híbrida: las cuestiones organizativas más urgentes e inmediatas se debatían de forma autónoma en la Red, mientras que las decisiones políticas de mayor calado se consensuaban de forma offline en reuniones abiertas con mayor número de mujeres y más separadas en el tiempo - una vez al mes-. Presentan, por tanto, un trabajo paralelo e interrelacionado en los ámbitos tradicionales y electrónicos a fin de lograr inmediatez sin renunciar a un discurso coherente, así como a formas de acción política efectivas (Juris, 2012; Núñez Puente, 2011a).

La escasa interacción con partidos políticos y sindicatos, el despliegue de lazos de apoyo con otros movimientos sociales, la expresión de las acciones civiles y del debate feminista en la Red, así como el encuadre esencialmente movilizador de los mensajes, son algunas de las características de la estrategia digital planteada por parte de la Asamblea de Madrid en su perfil de Twitter. Estas manifiestan una vocación transversal del movimiento (Mondé, 2018) a la par que un interés expreso por posicionarse como un sujeto político autónomo $e$ independiente de otros actores tradicionales que operan en el espacio público (Pavan y Mainardi, 2018). Estas fórmulas manifiestan una utilización de los canales de comunicación vía Internet adaptados a las necesidades y naturaleza del movimiento en un nuevo contexto de protestas y acciones colectivas mediadas tecnológicamente.

\section{Referencias bibliográficas}

BANet-Weiser, Sarah. Empowered: Popular Feminism and Popular Misogyny. Durham, Duke University Press, 2018.

BANET-WEISER, Sarah; MILTNER, Kate M. \#MasculinitySoFragile: Culture, structure, and networked misogyny. Feminist Media Studies, vol. 16, n. 1, 2016, pp. 171-174. DOI: 10.1080/14680777.2016.1120490. 
BoIX, Montserrat. Hackeando el patriarcado en la lucha contra la violencia hacia las mujeres. Filosofía y práctica de mujeres en red desde el ciberfeminismo social. Mujeres en red, 2006. [http://www.mujeresenred.net/spip.php?article880 - acceso el 02 sep 2020].

CAMMAERTS, Bart. Protest logics and the mediation opportunity structure. European Journal of Communication, vol. 27, n. 2, 2012, pp. 117134.

CASERO-RIPOLLÉS, Andreu. Estrategias y prácticas comunicativas del activismo político en las redes sociales en España. Historia y Comunicación Social, vol. 20, n. 2, 2015, pp. 533-548.

CASTElls, Manuel. Comunicación y poder. Madrid, Alianza, 2009.

CASTElls, Manuel. Redes de indignación y esperanza. Madrid, Alianza, 2012.

Chenou, Jean-Marie; CePedA-MÁsmela, Carolina. \#NiUnaMenos: Data activism from the Global South. Television and New Media, vol. 20, n. 4, 2019, pp. 396-411.

ClARK, Rosemary. "Hope in a hashtag": the discursive activism of \#WhyIStayed. Feminist Media Studies, vol. 16, n. 5, 2016, pp. 788804. DOI: 10.1080/14680777.2016.1138235.

CORNELISSEN, Gert; KARELAIA, Natalia; SOYER, Emre. Clicktivism or slacktivism? Impression management and moral licensing. En: CORNELISSEN, Gert; ReUTSKAJA, Elena; Valenzuela, Ana (eds.). E European Advances in Consumer Research Volume. Duluth, Association for Consumer Research, 2013, pp. 244-244.

DADER, José Luis; CAMPOS-DomíngUEZ, Eva. La búsqueda digital del voto. Cibercampañas electorales en España 2015-2016. Valencia, Tirant lo Blanch, 2017.

De Miguel Álvarez, Ana. El movimiento feminista y la construcción de marcos de interpretación. El caso de la violencia contra las mujeres. Revista Internacional de Sociología, vol. 61, n. 3, 2003, pp. 127-150.

Delgado, J. M. y GutiéRREZ, J. Métodos y técnicas cualitativas de investigación en Ciencias Sociales. Madrid, Síntesis, 1995.

EDWARDS, Lee; PHILIP, Fiona; GerRARD, Ysabel. Communicating feminist politics? The double-edged sword of using social media in a feminist 
organization. Feminist Media Studies, vol. 16, n. 1, 2019, pp. 1-18. DOI: 10.1080/14680777.2019.1599036.

ESLEN-ZIYA, Hande. Social media and Turkish feminism: New resources for social activism. Feminist Media Studies, vol. 65, n. 1, 2013, pp. 860-870. 10.1080/14680777.2013.838369.

GILL, Rosalind. Culture and subjectivity in neoliberal and postfeminist times. Subjectivity, n. 25, 2008, pp. 432-445. DOI: 10.1057/sub.2008.28.

GING, Debbie. Alphas, Betas, and Incels: Theorizing the masculinities of the manosphere. Men and Masculinities, vol. 22, n. 4, 2017, pp. 638657. DOI: $10.1080 / 14680777.2018 .1450351$.

GING, Debbie. Bros v. Hos: Postfeminism, anti-feminism and the toxic turn in digital gender politics. En: GING, Debbie; SIAPERA, Eugenia (eds.). Gender hate online. Understanding the new antifeminism. Cham, Palgrave Macmillan, 2019, pp. 45-67.

HaRAWAY, D. J. Ciencia, cyborgs y mujeres. La reinvención de la naturaleza. Madrid, Cátedra, 1991.

IGARTUA, J. J. Métodos cuantitativos de investigación en comunicación. Barcelona, Bosch, 2006.

JURIS, Jeffrey. S. Reflections on \#Occupy Everywhere: Social media, public space, and emerging logics of aggregation. American Ethnologist, vol. 39, n. 2, 2012, pp. 259-279. DOI: 10.1163/9789004324589021.

JURIS, Jeffrey S.; PEREIRA, Inés; FEIXA, Carles. La globalización alternativa y los "novísimos" movimientos sociales. Revista del Centro de Investigación de La Universidad La Salle, vol. 10, n. 37, 2012, pp. 23-37. DOI: 10.26457/recein.v10i37.107.

LIGHT, J. S. The Digital Landscape: New space for women? Gender, Place \& Culture, vol. 2, n. 2, 1995, pp. 133-146. DOI: $\underline{10.1080 / 09663699550021982 .}$.

MCCHESNeY, R. W. Desconexión digital. Cómo el capitalismo está poniendo a internet en contra de la democracia. Barcelona, El Viejo Topo, 2015.

MICó, Josep-Luís, \& CASERO-RIPOLLÉS, Andreu. Political activism online: organization and media relations in the case of $15 \mathrm{M}$ in Spain. 
Information, Communication \& Society, vol. 17, n. 7, 2014, pp. 858-871. DOI: 10.1080/1369118X.2013.830634.

MONDÉ, Geniece Crawford. \#BlackDontCrack: a content analysis of the aging Black woman in social media. Feminist Media Studies, vol. 18, n. 1, 2018, pp. 47-60. DOI: 10.1080/14680777.2018.1409972.

NúÑEZ PUENTE, Sonia. Activismo y colectivos en red: praxis feminista online y violencia de género. Asparkía, n. 22, 2011a, pp. 85-98. DOI: 10.6035/Asparkia.

NÚÑEZ PUENTE, Sonia. Feminist cyberactivism: Violence against women, internet politics, and Spanish feminist praxis online. Continuum, vol. 25, n. 3, 2011b, pp. 333-346. DOI: $\underline{10.1080 / 10304312.2011 .562964 .}$

NúÑEZ PUENTE, Sonia; D’ANTONIO MACEIRAS, Sergio; FERNÁNDEZ ROMERO, Diana. Twitter activism and ethical witnessing: Possibilities and challenges of feminist politics against gender-based violence. Social Science Computer Review, 2019, pp. 1-17. DOI: $\underline{10.1177 / 0894439319864898 .}$.

NúÑEz Puente, Sonia; VÁzQuez CuPEIRO, Susana; FERnÁNDEZ ROMERO, Diana. Ciberfeminismo contra la violencia de género: análisis del activismo online-offline y de la representación discursiva de la víctima. Estudios sobre el Mensaje Periodístico, vol. 22, n. 2, 2016, pp. 861877. DOI: 10.5209/ESMP.54240.

ORGAD, Shani; GILL, Rosalind. Safety valves for mediated female rage in the \#MeToo era. Feminist Media Studies, vol. 19, n. 4, 2019, pp. 596-603. DOI: 10.1080/14680777.2019.1609198.

PAVAN, Elenna; MAINARDI, Arianna. Striking, marching, tweeting: Studying how online networks change together with movements. Partecipazione e Conflitto, vol. 11, n. 2, 2018, pp. 394-422. DOI: 10.1285/i20356609v11i2p394.

RÚDÓLFSDÓTTIR, Annadís G.; JÓHANNSDÓTTIR, Ásta. Fuck patriarchy! An analysis of digital mainstream media discussion of the \#freethenipple activities in Iceland in March 2015. Feminism and Psychology, vol. 28, n. 1, 2018, pp. 133-151. DOI: 10.1177/0959353517715876.

SÁNCHEZ-DUARTE, José Manuel; FERNÁNDEZ-ROMERO, Diana. Subactivismo feminista y repertorios de acción colectiva digitales: 
prácticas ciberfeministas en Twitter. El Profesional de la Información, vol. 26, n. 5, 2017, pp. 894-902. DOI: 10.3145/epi.2017.sep.111.

SANDOVAl-AlmazAN, Rodrigo; GiL-GarCiA, J. Ramón. Towards cyberactivism 2.0? Understanding the use of social media and other information technologies for political activism and social movements. Government Information Quarterly, vol. 31, n. 3, 2014, pp. 365-378.

VALCÁRCEL, A. La memoria colectiva y los retos del feminismo. Santiago de Chile, CEPAL, 2001.

VAN ZOONEN, Liebest. Gendering the Internet. Claims, controversies and cultures. European Journal of Communication, vol. 17, n. 1, 2002, pp. 5-23. DOI: $10.1177 / 0267323102017001605$.

WAJCMAN, Judy. Feminist theories of technology. Cambridge Journal of Economics, vol. 34, n. 1, 2009, pp. 143-152. DOI: 10.1093/cje/ben057.

WAJCMAN, Judy. From women and technology to gendered technoscience. Information, Communication \& Society, vol. 10, n. 3, 2007, pp. 287-298. DOI: 10.1080/13691180701409770.

WIMMER, R. D. y DOMINICK, J. R. La investigación científica de los medios de comunicación: una introducción a sus métodos. Barcelona, Bosch, 1996.

YOUNG, I. M. Vida política y diferencia de grupo: una crítica del ideal de ciudadanía universal. En: CASTELLS, Carme (ed.). Perspectivas feministas en teoría política. Barcelona, Paidós Ibérica, 1996, pp. 99126.

ZAFRA, Remedios. Un cuarto propio conectado. Feminismo y creación desde la esfera público-privada online. Asparkía, n. 22, 2011, pp. 115-129. [http://hdl.handle.net/11441/26551 - acceso el 09 oct 2020]. 